\title{
Dissociating diatomic molecules in ultrafast and intense light
}

\author{
Christian Buth ${ }^{\mathrm{a}}$ \\ ${ }^{a}$ Theoretische Chemie, Physikalisch-Chemisches Institut, Ruprecht-Karls-Universität Heidelberg, \\ Im Neuenheimer Feld 229, 69120 Heidelberg, Germany
}

\begin{abstract}
'An $a b$ initio theory is devised for the quantum dynamics of molecules undergoing (multiple) ionization in ultrafast and intense light. Specifically, the intertwined problem of photoionization, radiative, and electronic transitions in the course of dissociation is addressed which arises, e.g., when molecules are exposed to XUV light or x rays from free electron lasers 'or attosecond light sources, but the approach is equally useful in optical strong-field physics. The coherent interaction of the molecule with the light in a specific charge state is also treated. I set out from an abstract formulation in terms 'of the quantum optical notion of system-reservoir interaction using a master equation in Lindblad form and analyze its short-time approximation. First, I express it in a direct sum rigged Hilbert space for an efficient solution with numerical methods for systems of differential equations. Second, I derive a treatment via quantum Monte Carlo wave packet (MCWP) propagation. The formalism is concretized to diatomic molecules in Born-Oppenheimer approximation whereby molecular rotation is disregarded. The numerical integration of the master equation is carried out with a suitably factored density matrix that exploits the locality of the Hamiltonian and the Lindblad superoperator with respect to the internuclear distance. The formulation of the MCWP for molecules requires a thorough analysis of the quantum jump process; namely, the dependence on the continuous distance renders a straight wave packet promotion useless and, instead, a projected outer product needs to be employed involving an integrated quantum jump operator.

Keywords: Master equation in Lindblad form, Quantum Monte Carlo algorithm, Dissociation of diatomic molecules, Ultrafast and intense light, Born-Oppenheimer approximation
\end{abstract}

\section{Introduction}

Understanding the interaction of molecules with ultrafast and intense light poses a formidable challenge: there is the coherent interaction of the molecular electronic states of a specific charge state, photoionization, radiative, and electronic transitions among charge states intertwined with the nuclear quantum dynamics of rotation, vibration, and dissociation. Seminal steps towards this issue were made experimentally by examining the ion yields and the kinetic energy release (KER) of nitrogen molecules $\left(\mathrm{N}_{2}\right)$ in $\mathrm{x}$ rays of the Linac Coherent Light Source (LCLS) free electron laser (FEL) [1, 2]. Theoretically, phenomenological models [3] and molecular rate equations [4] were devised to unravel the physics of this multiple time-scale problem. Recently, also a rate-equation description of the molecular populations [5, 6] combined with classical nuclear dynamics 7] was used.

Nuclear dynamics in molecules in several charge states is treated in a time-dependent view on quantum mechanics 8]. Foundational work on the interdependence of electronic decay and nuclear dynamics has been carried out in Refs. 9, 10, 11, 12, 13, 14, 15]. Even optical laser control

Email address: christian.buth@web.de (Christian Buth) URL: http://www.christianbuth.name (Christian Buth) of the dissociation of $\mathrm{N}_{2}^{2+}$ can be realized [16, 17]. When emitted photons and electrons are not considered, an open quantum-system approach based on a master equation in Lindblad form [18, 19, 20, 21, 22, 23] needs to be employed. Such a description has been developed for diatomic molecules in Refs. [24, 25, 26, 27, 28, 29, 30, 31] using Monte Carlo wave packet propagation (MCWP), a quantum Monte Carlo (QMC) formalism [32, 33, 34, 35, 22, 23. Related notions of a treatment of dissipative processes have been named quantum-jump and quantum-trajectory approach 35]. The KER of $\mathrm{H}_{2}, \mathrm{HD}$, and $\mathrm{D}_{2}$ molecules in strong optical fields [24, 25, 26, 27, 29, 30, 31] and of $\mathrm{O}_{2}$ in XUV light of the SPring- 8 Compact SASE Source (SCSS) FEL [28, 29, 36] were investigated and a description via incoherent ionization rates was shown to be sufficient. Likewise, Refs. [3, 4] indicate that also radiative and electronic decay can be described by incoherent rates. This reduces the intricacy of the problem because now only the coherent interaction with light and the nuclear quantum dynamics need to be fully accounted for. Such coherent effects manifest in photoexcitation with the ensuing quantum dynamics which has been studied for a long time, e.g., Ref. [37], and is described by the present formalism as well as a special case. Yet there are issues with the definition of the quantum jump operator in Refs. [24, 25, 26, 27, 28, 29, 30, 31] which lead to an incor- 
rect $\mathrm{MCWP}$ scheme there despite a convincing agreement between computations and experiment.

Solving the master equation is in several ways simplified by MCWP. First, a density matrix has a quadratic dependence on the number of basis states of the rigged Hilbert space [38, 39] employed while a wave packet has only a linear dependence. Second, the propagation of the density matrix is inseparable and needs to be carried out as a whole whereas the propagation of wave packets in MCWP can be performed in parallel. Specifically, if the system is initially in a probabilistic mixture, MCWP propagation can be done for sufficiently many starting wave packets to adequately sample the mixture 22]. Third, MCWP offers an insightful physical interpretation of the interaction of the system with light in contrast to the abstract density operator formalism. Namely, if the ejection of photons and electrons from the molecule can be measured by gedanken detectors, then MCWP describes the physical quantum jumping between states and not only represents a sophisticated theoretical device to solve the master equation 32, 33, 34, 35, 22, 24, 25, 26, 27, 28, 29, 30, 31.

The article is organized as follows. The master equation in Lindblad form is discussed abstractly in Sect. 2, first, in general [Sect. 2.1] and, second, in a short-time approximation [Sect.2.2. Assuming a system in various charge states in Sect. 2.3, the master equation is expressed in a direct sum [40] rigged Hilbert space [38, 39]. A discrete time quantum Monte Carlo algorithm is devised in Sect. 2.4. The formalism of sections 2 is concretized to dissociating molecules in Sect. 3. The direct sum rigged Hilbert space, the Hamiltonian, and the master equation are explicated in Sect. 3.1] Sect. 3.2, and Sect. 3.3, respectively. The density matrix is factored in Sect. 3.4 with respect to the internuclear distance and a system of partial differential equations (PDEs) is derived which is amenable to a solution with established numerical methods. The MCWP scheme is devised in Sect. 3.5 for an integrated quantum jump operator. Conclusions are drawn in Sect. 4. I formulate the theory for diatomic molecules which, however, can be generalized to larger molecules, e.g., by using multiconfiguration time-dependent Hartree (MCTDH) [41, 42, 37] which is a powerful approach to treat nuclear dynamics quantum mechanically for a specific charge state of a molecule. Atomic units are used throughout [43, 44].

\section{Master equation in Lindblad form}

\subsection{System-reservoir interaction}

The most general quantum mechanical description of a system is given by a density operator or probabilistic operator 1 which is denoted by $\hat{\rho}(t)$ for time $t[19,20,21,445$,

\footnotetext{
${ }^{1}$ The density operator is frequently also referred to as "statistical operator" which is a misnomer as the density operator models a random phenomenon and it is not used for the analysis of data from such a phenomenon.
}

22, 23, 46]. It facilitates to jointly describe pure quantum states and probabilistic mixtures where for the latter, insufficient information is available, i.e., the state of the system has not been fully characterized in terms of a complete experiment, and thus it cannot be expressed in terms of a single state vector. The behavior of $\hat{\rho}(t)$ is elucidated within the quantum optical notion of system-reservoir interaction [19, 20, 21, 22, 23] as theoretical framework. It is particularly useful, in my case of an open system, for which electrons and photons are ejected into the continuum, i.e., the reservoir, and thus particles and energy are dissipated. As the state of the reservoir remains undetected (there are only gedanken detectors), a single-state-vector representation is not feasible.

The time-dependent quantum dynamics of the system and the reservoir is described by the analog of the Schrödinger equation for the joint density operator which is termed the Liouville or von Neumann equation; it describes physical processes which are reversible in time [19, 20, 21, 22, 23]. The combined system and reservoir is a closed system; the master equation for the system only is derived by considering the system-reservoir coupling up to second order in perturbation theory. Allowing the reservoir to "loose its memory" by introducing the Markoff approximation - which is in this context equivalent to Weisskopf-Wigner theory [47] of decay processes [22] - results in irreversibility of a quantum process. This leads to an equation for the reduced density operator that comprises only the system reading

$$
\frac{\partial \hat{\rho}(t)}{\partial t}=-\mathrm{i}[\hat{H}, \hat{\rho}(t)]+\hat{\mathcal{L}}_{\mathrm{Ld}}[\hat{\rho}(t)]
$$

with the Hamiltonian $\hat{H}$ of the system. The bracket in the first term on the right-hand side of the equation denotes a commutator which accounts for the coherent evolution of the system; without system-reservoir interaction, this is the only term on the right-hand side and Eq. (1) has the form of a Liouville equation. The second term is the Liouvillian superoperator $\hat{\mathcal{L}}_{\mathrm{Ld}}[\hat{\rho}(t)]$ that describes the dissipation of electrons and photons into the continuum. Additional norm nonconserving terms arise, if not all final states of the system are incorporated because then transitions to states not included in the density operator cause a loss of norm. I assume that all final states are incorporated and thus the trace of the density operator is unity for all times. This property of $\hat{\mathcal{L}}_{\mathrm{Ld}}[\hat{\rho}(t)]$ implies that the Liouvillian has Lindblad form [18, 19, 20, 21, 22, 23]. It is expressed in terms of quantum jump operators

$$
\hat{J}_{\phi \psi}=\sqrt{\gamma_{\phi \psi}(t)}|\phi\rangle\langle\psi|,
$$

for $\phi, \psi \in \mathbb{B}$ with a basis $\mathbb{B}$ of the rigged Hilbert space [38, 39]. The transition rate from $|\psi\rangle$ to $|\phi\rangle$ is $\gamma_{\phi \psi}(t)$; it vanishes for $\phi=\psi$. Thus norm conserving relaxation processes [18, 19, 20, 21, 22, 23] are effected by

$$
\hat{\mathcal{L}}_{\mathrm{Ld}}[\hat{\rho}(t)]=\hat{\mathcal{L}}_{\mathrm{Nm}}[\hat{\rho}(t)]+\hat{\mathcal{L}}_{\mathrm{Jp}}[\hat{\rho}(t)]
$$




$$
\begin{aligned}
= & -\frac{1}{2} \sum_{\phi, \psi \in \mathbb{B}}\left[\hat{J}_{\phi \psi}^{\dagger} \hat{J}_{\phi \psi} \hat{\rho}(t)+\hat{\rho}(t) \hat{J}_{\phi \psi}^{\dagger} \hat{J}_{\phi \psi}\right] \\
& +\sum_{\phi, \psi \in \mathbb{B}} \hat{J}_{\phi \psi} \hat{\rho}(t) \hat{J}_{\phi \psi}^{\dagger} \\
= & -\frac{1}{2} \sum_{\psi \in \mathbb{B}} \Gamma_{\psi}(t)\left[\hat{\mathcal{P}}_{\psi} \hat{\rho}(t)+\hat{\rho}(t) \hat{\mathcal{P}}_{\psi}\right] \\
& +\sum_{\phi, \psi \in \mathbb{B}} \gamma_{\phi \psi}(t)\langle\psi|\hat{\rho}(t)| \psi\rangle \hat{\mathcal{P}}_{\phi} ;
\end{aligned}
$$

it consists of a norm reducing superoperator $\hat{\mathcal{L}}_{\mathrm{Nm}}[\hat{\rho}(t)]$ and a quantum jump superoperator $\hat{\mathcal{L}}_{\mathrm{Jp}}[\hat{\rho}(t)]$. The total transition rate is

$$
\Gamma_{\psi}(t)=\sum_{\phi \in \mathbb{B}} \gamma_{\phi \psi}(t) .
$$

The projector on a state vector $|\psi\rangle$ is $\hat{\mathcal{P}}_{\psi}=|\psi\rangle\langle\psi|$. In the brackets in Eq. (4) an anticommutator of $\hat{\rho}(t)$ with $\hat{\mathcal{P}}_{\psi}$ is spelled out.

The diagonal elements of the density matrix $p_{\phi}(t)=$ $\langle\phi|\hat{\rho}(t)| \phi\rangle$ are the populations - i.e., probabilities for discrete states and probability densities for continuum states - to find the system in the state $|\phi\rangle$ for $\phi \in \mathbb{B}$. Taking the diagonal elements of the master equation (1), (3), I obtain a Pauli master equation [48, 19, 20] —a rate equation-reading

$$
\begin{aligned}
\frac{\partial p_{\phi}(t)}{\partial t}= & -\mathrm{i}\langle\phi|[\hat{H}, \hat{\rho}(t)]| \phi\rangle+\sum_{\psi \in \mathbb{B}} \gamma_{\phi \psi}(t) p_{\psi}(t) \\
& -\Gamma_{\phi}(t) p_{\phi}(t) .
\end{aligned}
$$

The first term on the right-hand side describes the coherent time evolution of the system governed by $\hat{H}$ whereas the remaining summands are relaxation terms from system-reservoir interaction (3). Rate equations are frequently applied in the semiclassical theory of the laser [21, 22] to describe the absorption and stimulated emission of light. If $\hat{H}$ has a matrix representation that is diagonal with respect to $\mathbb{B}$, i.e., it does not couple basis states, the matrix element of the commutator in (6) vanishes. In this form, rate equations play an important role in the understanding of the ionization of atoms [3, 49, 50, 51] and molecules [3, 44 by intense light in the optical and x-ray regimes. The derivation of Eq. (6) from Eqs. (1), (3) represents an a posteriori justification of the rate-equation approximation of Refs. [3, 4, 49, 50, 51]. This, in turn, assures me that the present theory describes the interaction with the light adequately.

\subsection{Short-time approximation}

I recast the master equation (11) with the Lindblad superoperator (3) into the form [22] of

$$
\frac{\partial \hat{\rho}(t)}{\partial t}=-\mathrm{i}\left(\hat{H}_{\mathrm{eff}} \hat{\rho}(t)-\hat{\rho}(t) \hat{H}_{\text {eff }}^{\dagger}\right)+\hat{\mathcal{L}}_{\mathrm{Jp}}[\hat{\rho}(t)],
$$

with the effective Hamiltonian

$$
\hat{H}_{\text {eff }}=\hat{H}+\hat{V}_{\text {ra }},
$$

that comprises the Hermitian 45] Hamiltonian $\hat{H}=\hat{H}^{\dagger}$ for the system and the non-Hermitian decay operator for the transitions away from a state $|\psi\rangle$ that cause loss of population of this state with the rate $\Gamma_{\psi}(t)$ via

$$
\hat{V}_{\text {ra }}=-\frac{\mathrm{i}}{2} \sum_{\psi \in \mathbb{B}} \Gamma_{\psi}(t) \hat{\mathcal{P}}_{\psi} .
$$

Inserting the expression for $\hat{H}_{\text {eff }}$ from Eq. (8), (9) into Eq. (7), the sum of the superoperator for the coherent evolution $-\mathrm{i}[\hat{H}, \hat{\rho}(t)]$ and the norm-reducing superoperator $\hat{\mathcal{L}}_{\mathrm{Nm}}[\hat{\rho}(t)]$ are obtained such that only $\hat{\mathcal{L}}_{\mathrm{Jp}}[\hat{\rho}(t)]$ remains to be added to reproduce the master equation (11), (3).

The master equation (7) can be approximately integrated for a short time interval $\delta t$ by replacing the temporal derivative on the left-hand side of Eq. (7) by a difference quotient yielding

$$
\begin{aligned}
\hat{\rho}(t+\delta t)= & \hat{\rho}(t)-\mathrm{i} \delta t\left(\hat{H}_{\mathrm{eff}} \hat{\rho}(t)-\hat{\rho}(t) \hat{H}_{\mathrm{eff}}^{\dagger}\right) \\
& +\delta t \hat{\mathcal{L}}_{\mathrm{Jp}}[\hat{\rho}(t)]+O\left(\delta t^{2}\right) .
\end{aligned}
$$

The Landau symbol [52] big- $O$ indicates terms with $\delta t^{2}$ and higher orders. I refer to this equation as shorttime master equation. The separation into contributions from $\hat{H}_{\text {eff }}$ and contributions from $\hat{\mathcal{L}}_{\mathrm{Jp}}[\hat{\rho}(t)]$ is fundamental and a consequence of the linearization of the master equation with respect to time differences $\delta t$ by neglecting the terms $O\left(\delta t^{2}\right)$. The time interval $\delta t$ needs to be longer than the reservoir memory time such that the Markoff approximation can be made; but $\delta t$ must also be shorter than the system time scale such that there is no appreciable change in the system variables [22].

I find from Eq. (10) by taking the trace on both sides of the equation and using the linearity of the trace [19, 20, 45, 46] and the fact that the trace of the probabilistic operator is unity at all times, that the trace of the two terms before $O\left(\delta t^{2}\right)$ are the same in magnitude with opposite sign such that they cancel upon neglecting terms of $O\left(\delta t^{2}\right)$, i.e.,

$$
\begin{aligned}
\delta p(t) & =\mathrm{i} \delta t \operatorname{Tr}\left(\hat{H}_{\text {eff }} \hat{\rho}(t)-\hat{\rho}(t) \hat{H}_{\text {eff }}^{\dagger}\right) \\
& =\delta t \operatorname{Tr}\left(\hat{\mathcal{L}}_{\mathrm{Jp}}[\hat{\rho}(t)]\right)+O\left(\delta t^{2}\right) .
\end{aligned}
$$

The Eq. (11) describes the fact that the non-Hermitian terms in Eq. (8) reduce the norm of the wave packet with time, i.e., the probability to remain in a state decreases. The loss of norm in the course of the temporal evolution is only apparent because of quantum jumps to other states which counter the loss and cause the conservation of the trace of the probabilistic operator.

The density operator $\hat{\rho}(\tau)$ at the initial time $\tau$ describes a probabilistic mixture of states and is represented in terms of orthonormal state vectors $|\Psi, \tau\rangle$ for $\Psi \in \mathbb{S}$. The countable set $\mathbb{S} \subseteq \mathbb{B}$ is the initial-state composition of the density 
operator; for an initial state which is pure, $\mathbb{S}$ has only one element, i.e., the system is described by a single state vector. The probabilities to find the system in the respective state are $W_{\Psi}$. As remains to be shown, $\hat{\rho}(t)$ is diagonal at all times $t$ in terms of the time-evolving state vectors of $\mathbb{S}$. Let the density operator be diagonal at time $t$ [20, 22], i.e.,

$$
\hat{\rho}(t)=\sum_{\Psi \in \mathbb{S}}|\Psi, t\rangle W_{\Psi}\langle\Psi, t| .
$$

Specifically, the trace condition (11) yields - for the probability of the system to undergo a quantum jump in the time interval $\delta t,-$ by inserting Eq. (12), the expression

$$
\begin{aligned}
\delta p(t) & =\sum_{\Psi \in \mathbb{S}} W_{\Psi} \delta p_{\Psi}(t) \\
& =\mathrm{i} \delta t \sum_{\Psi \in \mathbb{S}} W_{\Psi}\left\langle\Psi, t\left|\hat{H}_{\mathrm{eff}}-\hat{H}_{\mathrm{eff}}^{\dagger}\right| \Psi, t\right\rangle \\
& =\delta t \sum_{\Psi \in \mathbb{S}} W_{\Psi} \sum_{\psi \in \mathbb{B}} \Gamma_{\psi}(t)|\langle\psi \mid \Psi, t\rangle|^{2},
\end{aligned}
$$

with the total decay rate (5) of $|\psi\rangle$.

Inserting Eq. (12) into Eq. (10), I can express the first and second terms on the right-hand side of Eq. (10), i.e., without the quantum jump contribution, in terms of the time evolution of a state vector 2 obeying

$$
|\tilde{\Psi}, t+\delta t\rangle=|\Psi, t\rangle-\mathrm{i} \delta t \hat{H}_{\mathrm{eff}}|\Psi, t\rangle+O\left(\delta t^{2}\right) .
$$

This is a short-time approximation of the temporal propagation of a wave packet [32, 33, 34, 35, 22] using the time-dependent Schrödinger equation [45] with the effective Hamiltonian (8), i.e.,

$$
\mathrm{i} \frac{\partial}{\partial t}|\Psi, t\rangle=\hat{H}_{\text {eff }}|\Psi, t\rangle \text {. }
$$

I use a tilde on $|\tilde{\Psi}, t+\delta t\rangle$ in Eq. (14) to indicate that-due to the non-Hermiticity of $\hat{H}_{\text {eff }}$-it is not normalized but

$$
\begin{aligned}
& \langle\tilde{\Psi}, t+\delta t \mid \tilde{\Psi}, t+\delta t\rangle \\
= & 1-\mathrm{i} \delta t\left\langle\Psi, t\left|\hat{H}_{\mathrm{eff}}-\hat{H}_{\mathrm{eff}}^{\dagger}\right| \Psi, t\right\rangle+O\left(\delta t^{2}\right) \\
= & 1-\delta p_{\Psi}(t)+O\left(\delta t^{2}\right),
\end{aligned}
$$

holds with (13). Likewise inserting Eq. (12) into Eq. (10), I express the third term on the right-hand side as a double sum over the outer product of $\sqrt{\delta t} \hat{J}_{\phi \psi}|\Psi, t\rangle$. This state is also not normalized but I have

$$
\delta p_{\Psi}(t)=\delta t \sum_{\phi, \psi \in \mathbb{B}}\left\langle\Psi, t\left|\hat{J}_{\phi \psi}^{\dagger} \hat{J}_{\phi \psi}\right| \Psi, t\right\rangle
$$

with Eqs. (2) and (13).

I rewrite the short-time master equation (10) based on probabilities 22] using Eqs. (12), (14), (16), and (17) arriving at

$$
\begin{aligned}
\hat{\rho}(t+\delta t)= & \sum_{\Psi \in \mathbb{S}} W_{\Psi}\left(1-\delta p_{\Psi}(t)\right) \frac{|\tilde{\Psi}, t+\delta t\rangle}{\sqrt{1-\delta p_{\Psi}(t)}} \frac{\langle\tilde{\Psi}, t+\delta t|}{\sqrt{1-\delta p_{\Psi}(t)}} \\
& +\sum_{\Psi \in \mathbb{S}} W_{\Psi} \delta p_{\Psi}(t) \sum_{\phi, \psi \in \mathbb{B}} \sqrt{\frac{\delta t}{\delta p_{\Psi}(t)}} \hat{J}_{\phi \psi}|\Psi, t\rangle\langle\Psi, t| \hat{J}_{\phi \psi}^{\dagger} \sqrt{\frac{\delta t}{\delta p_{\Psi}(t)}}+O\left(\delta t^{2}\right) .
\end{aligned}
$$

The probabilistic operator at time $t+\delta t$ is diagonal with respect to the new states; it is composed of a nonHermitian propagation from the first and second terms on the right-hand side of Eq. (10) and a quantum-jump contribution from the third term in Eq. (10) which are multiplied by the remaining norm $1-\delta p_{\Psi}(t)[\mathrm{Eq}$. (16) $]$ and the loss of norm $\delta p_{\Psi}(t)$ [Eq. [17)], respectively.

\subsection{Numerical integration}

The master equation (7) is a system of coupled differential equations which are first-order in the time derivative.

\footnotetext{
${ }^{2}$ In stating Eq. (14), I do not presuppose that the temporal evolution is given by $|\tilde{\Psi}, t+\delta t\rangle=\mathrm{e}^{-\mathrm{i} \hat{H}_{\text {eff }} \delta t}|\Psi, t\rangle$ which is only fulfilled for a time-independent $\hat{H}_{\text {eff }}$.
}

The short-time master equation (10) has the form of a numerical scheme for a discretized time upon neglecting terms $O\left(\delta t^{2}\right)$ and can be used right away. This is actually the Euler method [53] in the case that the Hamiltonian does not contain any differential operators and thus (7) is a system of first-order ordinary differential equations. In practice, however, one would not employ the Euler method [53] but rather use, e.g., the more accurate RungeKutta method of fourth order 54] as done in Ref. [55] to integrate the Liouville equation for the density operator of molecules aligned by an intense optical laser.

Although a method for solving (7) is introduced in the previous paragraph, further simplifications can be achieved by making more assumptions about the context 
which is here photoionization, radiative, and electronic transitions whereby the photons and electrons liberated in transitions induced by the Lindblad superoperator are disregarded. Let the neutral system have $N \in \mathbb{N}$ electrons and let the maximum charge state considered be $N_{\mathrm{el}} \in \mathbb{N}_{0}$, $N_{\mathrm{el}} \leq N$. Then the states in $\mathbb{B}$ are a basis of the direct sum [40] of the $n$-electron rigged Hilbert spaces [38, 39] for $n \in\left\{N-N_{\mathrm{el}}, \ldots, N\right\}=\mathbb{A}_{\mathrm{el}}$. The equations of the previous Sect. 2 are formulated in this space. The Hamilton operator decomposes $\hat{H}=\sum_{n \in \mathbb{A}_{\mathrm{el}}} \hat{H}^{(n)}$ into a sum of Hamiltonians $\hat{H}^{(n)}$ for charge state $n$. Looking at $\hat{V}_{\text {ra }}$ [Eq. (9)], I realize, that the sum/integral therein can be partitioned such that $\hat{H}_{\text {eff }}=\sum_{n \in \mathbb{A}_{\mathrm{el}}} \hat{H}_{\text {eff }}^{(n)}$ holds with (8). Let $\hat{\mathfrak{P}}^{(n)}$ be the projection operator onto the $n$-electron rigged Hilbert space and $\hat{\mathbb{1}}=\sum_{n \in \mathbb{A}_{\mathrm{el}}} \hat{\mathfrak{P}}^{(n)}$. Looking at Eq. (3) , I find that $\hat{\mathcal{L}}_{\mathrm{Jp}}[\hat{\rho}(t)]=\sum_{n \in \mathbb{A}_{\mathrm{el}}} \hat{\mathfrak{P}}^{(n)} \hat{\mathcal{L}}_{\mathrm{Jp}}[\hat{\rho}(t)] \hat{\mathfrak{P}}^{(n)}$

The master equation (7) in the $n$-electron rigged Hilbert space [38, 39] is obtained by projecting it onto $\hat{\mathfrak{P}}^{(n)}$ for $n \in$ $\mathbb{A}_{\mathrm{el}}$ writing

$$
\begin{aligned}
\frac{\partial \hat{\rho}^{(n)}(t)}{\partial t}= & -\mathrm{i}\left(\hat{H}_{\mathrm{eff}}^{(n)} \hat{\rho}^{(n)}(t)-\hat{\rho}^{(n)}(t) \hat{H}_{\mathrm{eff}}^{(n) \dagger}\right) \\
& +\hat{\mathfrak{P}}^{(n)} \hat{\mathcal{L}}_{\mathrm{Jp}}[\hat{\rho}(t)] \hat{\mathfrak{P}}^{(n)}
\end{aligned}
$$

with $\hat{\rho}^{(n)}(t)=\hat{\mathfrak{P}}^{(n)} \hat{\rho}(t) \hat{\mathfrak{P}}^{(n)}$. This implies that

$$
\hat{\rho}(t)=\sum_{n \in \mathbb{A}_{\mathrm{el}}} \hat{\rho}^{(n)}(t),
$$

holds for all times $t \geq \tau$ where at the initial time $\tau$ the system is neutral, i.e., $\hat{\rho}(\tau)=\hat{\rho}^{(N)}(\tau)$. Let $\hat{\mathcal{L}}_{\mathrm{Jp}_{\mathrm{p}}}[\hat{\rho}(t)]$ exclusively describe quantum jumps to the same charge state or to higher charge states, i.e.,

$$
\hat{\mathfrak{P}}^{(n)} \hat{\mathcal{L}}_{\mathrm{Jp}}[\hat{\rho}(t)] \hat{\mathfrak{P}}^{(n)}=\sum_{m=n}^{N} \hat{\mathfrak{P}}^{(n)} \hat{\mathcal{L}}_{\mathrm{Jp}}\left[\hat{\rho}^{(m)}(t)\right] \hat{\mathfrak{P}}^{(n)} .
$$

With this, Eq. (19) for $n=N$ can be solved independently of higher charge states $n<N$ because $\hat{\mathfrak{P}}^{(N)} \hat{\mathcal{L}}_{\mathrm{Jp}}[\hat{\rho}(t)] \hat{\mathfrak{P}}^{(N)}=\hat{\mathfrak{P}}^{(N)} \hat{\mathcal{L}}_{\mathrm{Jp}}\left[\hat{\rho}^{(N)}(t)\right] \hat{\mathfrak{P}}^{(N)}$ provided that $\hat{\rho}^{(N)}(\tau)$ is given at the initial time $\tau$. For $n<N$, there are terms which couple among rigged Hilbert spaces with the same or higher electron number. The number of charge states involved depends on the processes considered, apart from radiative decay and the coherent interaction in a charge state. For photoionization and Auger decay, only the charge state with the next higher electron number is involved. In the cases that two-electron emission, e.g., due to photoionization shake off or double Auger decay [49, 50], are taken into account, also the charge state with the second next higher electron number enters Eq. (19) for $n \leq N-2$. The hierarchy of projected master equations (19) is solved successively with numerical methods for differential equations.

\subsection{Random sampling}

The numerical integration of the master equation [Sect. 2.3 may become prohibitively expensive. Therefore, I formulate a quantum Monte Carlo (QMC) algorithm for the solution of the master equation (7) which also is referred to as random sampling or Monte Carlo wave packets (MCWPs) [32, 33, 34, 35, 22]. Namely, the system of differential equations for the density operator (7) has number of basis states squared, i.e., $|\mathbb{B}|^{2}$, many equations, if no further assumptions are made. Based on the favorable form of Eq. (18), quantum trajectories 32, 33, 34, 35, 22. can be constructed. This reduces the task of solving the master equation (7) to the time propagation and quantumjumping of state vectors.

\subsubsection{Quantum trajectories}

To construct $|\mathbb{T}|$ many quantum trajectories, with indices in the set $\mathbb{T} \subseteq \mathbb{N}$, I start from the mixture of state vectors $\mathbb{S}$ at the initial time $\tau$ with a diagonal probabilistic operator (12). These initial states are the same in all trajectories $j \in \mathbb{T}$. The state vector $\left|\Psi_{j}, t\right\rangle$ for $\Psi_{j} \in \mathbb{S}$ at time $t \geq \tau$ evolves into a state vector at time $t+\delta t$, i.e., the next element in all $\mathbb{T}$ trajectories, by the following two rules:

First, non-Hermitian propagation (14): with probability $1-\delta p_{\Psi_{j}}(t)[\mathrm{Eq}$. [16)], the state vector at time $t$ is succeeded at time $t+\delta t$ by

$$
\left|\Psi_{j}, t\right\rangle \rightarrow \frac{\left|\tilde{\Psi}_{j}, t+\delta t\right\rangle}{\sqrt{1-\delta p_{\Psi_{j}}(t)}} .
$$

Second, quantum jumps [last term in Eq. (18)]: with probability $\delta p_{\Psi_{j}}(t)$ [Eq. (17)] the state vector $\left|\Psi_{j}, t\right\rangle$ at time $t$ is transformed into a different state vector at time $t+\delta t$. For the transition $|\psi\rangle \rightarrow|\phi\rangle$, this occurs via

$$
\left|\Psi_{j}, t\right\rangle \rightarrow \sqrt{\frac{\delta p_{\Psi_{j}}(t)}{\delta p_{\Psi_{j}}^{\phi \psi}(t)}} \sqrt{\frac{\delta t}{\delta p_{\Psi_{j}}(t)}} \hat{J}_{\phi \psi}\left|\Psi_{j}, t\right\rangle
$$

with the normalization $\sqrt{\frac{\delta p_{\Psi_{j}}(t)}{\delta p_{\Psi_{j}}^{\phi \psi}(t)}}$ and $\delta p_{\Psi_{j}}^{\phi \psi}(t) \neq 0$; otherwise no such jump occurs. The transition probability from where to where quantum jumps occur follows from the last line of Eq. (13), and Eqs. (2) and (17) to $\delta p_{\Psi_{j}}(t)=\underset{\phi, \psi \in \mathbb{B}}{\mathscr{\&}} \delta p_{\Psi_{j}}^{\phi \psi}(t)$. This gives the prescription

$$
\underbrace{}_{\phi, \psi \in \mathbb{B}} \frac{\delta p_{\Psi_{j}}^{\phi \psi}(t)}{\delta p_{\Psi_{j}}(t)}=1
$$

for the conditional probability/probability density [56] of a quantum jump $|\psi\rangle \rightarrow|\phi\rangle$ to occur to $\frac{\delta p_{\Psi_{j}}^{\phi \psi}(t)}{\delta p_{\Psi_{j}}(t)}$, if it is known that a quantum jump takes place.

To evaluate the master equation (18), I average over quantum trajectories in conjunction with summing over 
the probabilistic mixture which yields the density operator via

$$
\hat{\rho}(t+\delta t)=\lim _{|\mathbb{T}| \rightarrow \infty} \frac{1}{|\mathbb{T}|} \sum_{j \in \mathbb{T}} \sum_{\Psi_{j} \in \mathbb{S}}\left|\Psi_{j}, t+\delta t\right\rangle W_{\Psi_{j}}\left\langle\Psi_{j}, t+\delta t\right| .
$$

As Eq. (25) is diagonal for $t+\delta t$ it thus is for all times $t \geq \tau$.

\subsubsection{Quantum Monte Carlo algorithm}

Let $\mathbb{B}, \mathbb{S}$, and $\mathbb{T}$ be finite. The QMC algorithm [57] to calculate the density operator (25) with $\mathbb{T}$ quantum trajectories proceeds along the following four step 3 for each trajectory $j \in \mathbb{T}$.

First, choose the state vector $\Psi_{j} \in \mathbb{S}$ by drawing a uniformly distributed random number $r \in[0 ; 1[$; if $r$ falls into the partition

$$
\sum_{k=1}^{m-1} W_{\Psi^{(k)}} \leq r<\sum_{k=1}^{m} W_{\Psi^{(k)}}
$$

for an $m \in\{1, \ldots,|\mathbb{S}|\}$ where the states in $\mathbb{S}$ form the sequence $\Psi^{(k)} \in \mathbb{S}$ for $k \in\{1, \ldots,|\mathbb{S}|\}$, and an empty sum is zero, then the state vector $\Psi_{j}=\Psi^{(m)}$ is the initial state of quantum trajectory $j$ at time $\tau$.

Second, decide with the probability to quantum jump $\delta p_{\Psi_{j}}(t)$ [Eqs. (13), (17)] and a uniformly distributed random number $r^{\prime} \in[0 ; 1]$, whether to go to the third step for $r^{\prime} \in\left[0 ; 1-\delta p_{\Psi_{j}}(t)[\right.$, or, otherwise, to the fourth step.

Third, temporally propagate (22) the wave packet using Eq. (14) without terms $O\left(\delta t^{2}\right)$. If the end time of the propagation has not been reached yet, go to the second step replacing $t$ by $t+\delta t$; otherwise stop.

Fourth, quantum jump (23) the wave packet. There are $N_{\mathrm{I}} \in \mathbb{N}_{0}$ basis states $\psi \in \mathbb{B}$ for which $\left\langle\psi \mid \Psi_{j}, t\right\rangle \neq 0$ holds; they are enumerated as $\psi^{(\ell)}$ with $\ell \in\left\{1, \ldots, N_{\mathrm{I}}\right\}$. From these originating states, there are $N_{\mathrm{F}} \in \mathbb{N}_{0}$ basis states $\phi \in \mathbb{B}$ to which a quantum jump goes, i.e., $\exists \ell \gamma_{\phi \psi^{(\ell)}}(t) \neq 0$ [Eq. (2)]; I enumerate them as $\phi^{(k)}$ with $k \in\left\{1, \ldots, N_{\mathrm{F}}\right\}$. The pairs of indices of possible quantum jumps are arranged in the set

$$
\begin{gathered}
\mathbb{P}=\left\{(k, \ell) \mid k, \ell \in \mathbb{N} \wedge 1 \leq k \leq N_{\mathrm{F}} \wedge 1 \leq \ell \leq N_{\mathrm{I}}\right. \\
\left.\wedge \gamma_{\phi^{(k)} \psi^{(\ell)}}(t) \neq 0\right\} .
\end{gathered}
$$

The set $\mathbb{P}$ is totally ordered [58] under " $\leq$ " defined for $(k, \ell),\left(k^{\prime}, \ell^{\prime}\right) \in \mathbb{P}$ by $(k, \ell) \leq\left(k^{\prime}, \ell^{\prime}\right): \Longleftrightarrow k N_{\mathrm{F}}+\ell \leq$ $k^{\prime} N_{\mathrm{F}}+\ell^{\prime}$. The quantum jump $(k, \ell) \in \mathbb{P}$ takes place, if the uniformly distributed random number $r^{\prime \prime} \in[0 ; 1[$ lies

\footnotetext{
${ }^{3}$ This constitutes a modified algorithm [57] over what is employed in $24,25,26,27,28,29,30,31]$ in which the wave packet is always propagated whether a quantum jump occurs or not. Namely, it is decided in the second step whether to propagate or to quantum jump. This rearrangement over Refs. [24, 25, 26, 27, 28, 29, 30, 31] is possible because the loss of norm of the non-Hermitian propagation (16) can be determined with the state vector at time $t$ via Eq. (13) and thus the state vector at time $t+\delta t$ is not required.
}

in the interval

$$
\sum_{\substack{\left(k^{\prime}, \ell^{\prime}\right) \in \mathbb{P} \\\left(k^{\prime}, \ell^{\prime}\right)<(k, \ell)}} \frac{\delta p_{\Psi_{j}}^{\phi^{\left(k^{\prime}\right)} \psi^{\left(\ell^{\prime}\right)}}(t)}{\delta p_{\Psi_{j}}(t)} \leq r^{\prime \prime}<\sum_{\substack{\left(k^{\prime}, \ell^{\prime}\right) \in \mathbb{P} \\\left(k^{\prime}, \ell^{\prime}\right) \leq(k, \ell)}} \frac{\delta p_{\Psi_{j}}^{\phi^{\left(k^{\prime}\right)} \psi^{\left(\ell^{\prime}\right)}}(t)}{\delta p_{\Psi_{j}}(t)} .
$$

If the end time of the propagation has not been reached yet, go to the second step replacing $t$ by $t+\delta t$; otherwise stop.

Due to the first step above, the sum over $\Psi_{j} \in \mathbb{S}$ in Eq. (25) is not required and $\hat{\rho}(t) \approx \frac{1}{|\mathbb{T}|} \sum_{j \in \mathbb{T}}\left|\Psi_{j}, t\right\rangle\left\langle\Psi_{j}, t\right|$ for all $t \geq \tau$.

\section{Dissociating diatomic molecules}

I devise a theory for the nuclear dynamics of a diatomic molecule in ultrafast and intense light that induces (multiple) ionization. For this purpose, I concretize the abstract formalism of Sect. 2. Splitting of energy levels due to molecular rotation is assumed unresolved and I do not consider in detail rotational motion. In doing so, I acknowledge the fact that such motion takes place on a much longer, picosecond, time scale compared with electronic transitions and molecular distortion regarded here [59, 60]. Specifically, I do not account for excitation of molecular rotations due to electronic transitions or nuclear distortion and I disregard any influence on the rotational states by the light.

\subsection{Rigged Hilbert space and basis states}

The solution of the stationary many-electron Schrödinger equation of a molecule - with all degrees of freedom of the electrons and the nuclei included-to determine the ground state and excited states is a formidable task [43, 61]. Fortunately, in many cases, the coupling of the motion of the electrons and the nuclei in a molecule may be neglected because electrons are much lighter than nuclei and thus the movement of the electrons in thermal equilibrium is much faster than that of the nuclei such that the nuclei can be treated as fixed, if only the electronic structure is to be found. This leads to a separation of the total Hamiltonian into an electronic Hamiltonian which depends only parametrically on the nuclear coordinates and a nuclear Hamiltonian which contains an electronic potential that averages over the electronic coordinates and is called Born-Oppenheimer approximation (BOA) 62, 43, 8]; it is assumed throughout. In BOA, the eigenstates of the stationary Schrödinger equation with the electronic Hamiltonian form potential energy surfaces (PES) with respect to the nuclear coordinates [43, 8]; they include also the Coulomb repulsion energy between the nuclei in their arrangement; the adiabatic PES are used [8]. Thereby, I assume that there is a countable (finite in practice) number of electronic states which are square 
integrable, i.e., states in a Hilbert space (without rigging) which can be achieved by box normalization [45] or, typically, by expanding the molecular electronic wave functions in terms of a square-integrable Gaussian basis set [43]. Yet there are effects beyond the BOA [63, 61, 8], not treated here, which shall become relevant for highly excited molecules.

The quantum dynamics of the molecule takes place in the direct sum rigged Hilbert space introduced abstractly in Sect. 2.3. The space is spanned, as the first component, by the electronic basis states $|p ; m R\rangle_{\mathrm{E}}$ from the $p$-electron Hilbert space for $p \in \mathbb{A}_{\mathrm{el}}$ where the subscript "E" means that the ket involves the electronic coordinates. The electronic states are enumerated by $m \in$ $\left\{1, \ldots, N_{\mathrm{st}}^{(p)}\right\}=\mathbb{A}_{\mathrm{st}}^{(p)}$ where the number of states considered is $N_{\mathrm{st}}^{(p)} \in \mathbb{N}$. The dependence of the basis states on the internuclear distance $R \in] 0 ; \infty$ [ is only parametrically [43]. As a second component, I have the eigenstates of the position operator in terms of the internuclear distance $|R\rangle_{\mathrm{N}}$ where the subscript "N" indicates that the ket is formed with respect to the nuclear coordinate space. In total, I have the electronic-distortional basis states given by the tensor product [46] of both components for which orthogonality

${ }_{\mathrm{E}}\left\langle p ; m R\left|\otimes{ }_{\mathrm{N}}\left\langle R \mid q ; n R^{\prime}\right\rangle_{\mathrm{E}} \otimes\right| R^{\prime}\right\rangle_{\mathrm{N}}=\delta\left(R-R^{\prime}\right) \delta_{p q} \delta_{m n}$

holds. Here $\delta\left(R-R^{\prime}\right)$ stands for the Dirac- $\delta$ distribution and $\delta_{p q}$ is the Kronecker symbol [56]. There is the completeness relation

$$
\sum_{p \in \mathbb{A}_{\mathrm{el}}} \sum_{\ell \in \mathbb{A}_{\mathrm{st}}^{(p)}} \int_{0}^{\infty}|p ; \ell R\rangle_{\mathrm{E}} \otimes|R\rangle_{\mathrm{N} \mathrm{E}}\langle p ; \ell R| \otimes_{\mathrm{N}}\langle R| \mathrm{d} R=\hat{\mathbb{1}} .
$$

A detail concerns the electron-bare molecule which obviously does not have electronic states. The internuclear repulsion is described by Coulomb's law. The corresponding state is denoted as $|0 ; 1 R\rangle_{\mathrm{E}}$; it is the vacuum (no electron coordinates); the space are the complex numbers $\mathbb{C}[64]$. The parametric dependence on $R$ is only included for consistency with the notation for the other states; there is none in reality.

\subsection{Nuclear Hamilton operator}

The nuclear Hamiltonian that governs the quantum dynamics of the diatomic molecule in light is

$$
\hat{H}=\int_{0}^{\infty}\left(\hat{T}_{\mathrm{di}}+\hat{V}_{\mathrm{el}}+\hat{V}_{\mathrm{li}}\right) \otimes|R\rangle_{\mathrm{N} \mathrm{N}}\langle R| \mathrm{d} R
$$

it consists of the nuclear kinetic energy due to distortional motion $\hat{T}_{\mathrm{di}}$, the electronic energy $\hat{V}_{\mathrm{el}}$, and the coherent interaction of the molecule with light $\hat{V}_{\text {li }}$.

For a diatomic molecule, the nuclear kinetic energy is simplified by going into the center of mass reference frame.
Then the two-atom problem is turned into an effective oneatom problem [60] with the reduced mass $\mu$ giving

$$
-\frac{1}{2 \mu} \vec{\nabla}^{2}=-\frac{1}{2 \mu R} \frac{\partial^{2}}{\partial R^{2}} R+B(R) \hat{\vec{J}}^{2},
$$

in spherical polar coordinates [56] with the internuclear distance $R$. The angular momentum operator $\hat{\vec{J}}$ in Eq. (32) describes molecular rotation with the principal rotational constant [59, 60] given by $B(R)=\frac{1}{2 \mu R^{2}}$. The $R$ dependence of $B(R)$ in Eq. (32) causes a coupling of rotational and distortional motion [59]. I neglect rotational degrees of freedom and write for the nuclear kinetic energy due to distortional motion

$$
\hat{T}_{\mathrm{di}}=\sum_{p \in \mathbb{A}_{\mathrm{el}}} \sum_{m \in \mathbb{A}_{\mathrm{st}}^{(p)}}|p ; m R\rangle_{\mathrm{E}}\left(-\frac{1}{2 \mu R} \frac{\partial^{2}}{\partial R^{2}} R\right)^{\prime}{ }_{\mathrm{E}}\langle p ; m R| .
$$

The prime on the differential operator indicates that derivatives of the electronic states with respect to $R$ are omitted, i.e., the BOA; the neglected terms are also local in the internuclear distance [8].

The electronic energy $\hat{V}_{\text {el }}$ in Eq. (31) contains all PESs and can be expressed succinctly by

$$
\hat{V}_{\mathrm{el}}=\sum_{p \in \mathbb{A}_{\mathrm{el}}} \sum_{m \in \mathbb{A}_{\mathrm{st}}^{(p)}}|p ; m R\rangle_{\mathrm{E}} E_{m}^{(p)}(R)_{\mathrm{E}}\langle p ; m R|
$$

where the PESs are given by $E_{m}^{(p)}(R)$.

The coherent interaction of the molecule with light $\hat{V}_{\text {li }}$ in Eq. (31) is treated semiclassically by

$$
\hat{V}_{\mathrm{li}}=\sum_{p \in \mathbb{A}_{\mathrm{el}}} \sum_{m, n \in \mathbb{A}_{\mathrm{st}}^{(p)}}|p ; m R\rangle_{\mathrm{E}} \wp_{m n}^{(p)}(R, t)_{\mathrm{E}}\langle p ; n R|
$$

Here $\wp_{m n}^{(p)}(R, t)$ is the interaction matrix element with the light that depends on its vector potential $\vec{A}(t)$ [45] which is given in the laboratory-fixed reference frame. The polar $\vartheta$ and azimuth $\varphi$ angles [56] specify the orientation of the internuclear axis in the laboratory-fixed reference frame. With the Euler matrix $\boldsymbol{R}_{\mathrm{E}}(\varphi, \vartheta, 0)$ [59] I transform $\vec{A}(t)$ from the laboratory-fixed frame to the molecule-fixed frame $\boldsymbol{R}_{\mathrm{E}}(\varphi, \vartheta, 0) \vec{A}(t)$ (see also Sec. II B 1 of Ref. [55]).

\subsection{Master equation in Lindblad form}

The abstract equations of Sect. 2 are rewritten for diatomic molecules by making the replacements

$$
\begin{aligned}
& |\psi\rangle \longrightarrow|p ; m R\rangle_{\mathrm{E}} \otimes|R\rangle_{\mathrm{N}} \\
& \langle\psi| \longrightarrow \mathrm{E}\langle p ; m R| \otimes{ }_{\mathrm{N}}\langle R| \\
& \underbrace{}_{\psi \in \mathbb{B}} \longrightarrow \sum_{p \in \mathbb{A}_{\mathrm{el}}} \sum_{m \in \mathbb{A}_{\mathrm{st}}^{(p)}} \int_{0}^{\infty} \mathrm{d} R \\
& \hat{\mathcal{P}}_{\psi} \longrightarrow \hat{\mathcal{P}}_{m}^{(p)}(R)
\end{aligned}
$$




$$
\begin{aligned}
& \gamma_{\phi \psi}(t) \longrightarrow \delta\left(R-R^{\prime}\right) \gamma_{n m}^{(q p)}(R, t) \\
& \Gamma_{\psi}(t) \longrightarrow \Gamma_{m}^{(p)}(R, t)
\end{aligned}
$$

The probabilistic operator in terms of the electronicdistortional states (29) reads

$$
\begin{aligned}
\hat{\rho}(t)= & \sum_{p \in \mathbb{A}_{\mathrm{el}}} \sum_{m, n \in \mathbb{A}_{\mathrm{st}}^{(p)}} \int_{0}^{\infty} \int_{0}^{\infty}|p ; m R\rangle_{\mathrm{E}} \otimes|R\rangle_{\mathrm{N}} \\
& \times \rho_{m n}^{(p)}\left(R, R^{\prime}, t\right)_{\mathrm{E}}\left\langle p ; n R^{\prime}\right| \otimes_{\mathrm{N}}\left\langle R^{\prime}\right| \mathrm{d} R \mathrm{~d} R^{\prime},
\end{aligned}
$$

with the density matrix $\rho_{m n}^{(p)}\left(R, R^{\prime}, t\right)$ that accounts for the form (20). The master equation (1) contains incoherent transitions by photoionization and spontaneous radiative and electronic decay in $\hat{\mathcal{L}}_{\mathrm{Ld}}[\hat{\rho}(t)]$ [Eq. (3) $]$. Hence decoherence results because the emitted photons and electrons are unobserved which was the initial motivation to use a density operator. Apart from spontaneous radiative decay, transitions between states with the same molecular charge are not described by $\hat{\mathcal{L}}_{\mathrm{Ld}}[\hat{\rho}(t)]$ [Eq. (3) ]. Instead, such transitions between PES are treated fully coherently by $\hat{H}$ [Eq. (31)] and thus the quantum mechanical phases are included.

The Lindblad operator (3) is expressed for diatomic molecules with the projection operator $\hat{\mathcal{P}}_{n}^{(q)}(R)$ and the quantum jump operator [Eq. (2)] reading

$\hat{J}_{n m}^{(q p)}(R)=\sqrt{\gamma_{n m}^{(q p)}(R, t)}|q ; n R\rangle_{\mathrm{E}} \otimes|R\rangle_{\mathrm{N} \mathrm{E}}\langle p ; m R| \otimes_{\mathrm{N}}\langle R|$.

Note that a $\delta\left(R-R^{\prime}\right)$ factor arising from the replacements (36) applied to (2) is not included in the defintion (38). The specific form of (38) implies vertical transitions for which the internuclear distance of the initial and the final state is the same. Consequently, the nonHermitian Hamiltonian (9) for the transitions away from a state $|p ; m R\rangle_{\mathrm{E}} \otimes|R\rangle_{\mathrm{N}}$ that cause a loss of population of this state with the rate $\Gamma_{n}^{(p)}(R, t)$ becomes

$$
\hat{V}_{\mathrm{ra}}=-\frac{\mathrm{i}}{2} \sum_{p \in \mathbb{A}_{\mathrm{el}}} \sum_{m \in \mathbb{A}_{\mathrm{st}}^{(p)}} \int_{0}^{\infty} \Gamma_{m}^{(p)}(R, t) \hat{\mathcal{P}}_{m}^{(p)}(R) \mathrm{d} R,
$$

where the total transition rate (5) is

$$
\Gamma_{m}^{(p)}(R, t)=\sum_{q \in \mathbb{A}_{\mathrm{el}}} \sum_{n \in \mathbb{A}_{\mathrm{st}}^{(q)}} \gamma_{n m}^{(q p)}(R, t)
$$

The quantum jump superoperator (3) reads

$$
\begin{aligned}
\hat{\mathcal{L}}_{\mathrm{Jp}}[\hat{\rho}(t)] & =\sum_{p, q \in \mathbb{A}_{\mathrm{el}}} \sum_{m \in \mathbb{A}_{\mathrm{st}}^{(p)}} \sum_{n \in \mathbb{A}_{\mathrm{st}}^{(q)}} \int_{0}^{\infty} \hat{J}_{n m}^{(q p)}(R) \hat{\rho}(t) \hat{J}_{n m}^{(q p) \dagger}(R) \mathrm{d} R \\
& =\sum_{p, q \in \mathbb{A}_{\mathrm{el}}} \sum_{m \in \mathbb{A}_{\mathrm{st}}^{(p)}} \sum_{n \in \mathbb{A}_{\mathrm{st}}^{(q)}} \int_{0}^{\infty} \gamma_{n m}^{(q p)}(R, t) \rho_{m m}^{(p)}(R, R, t) \hat{\mathcal{P}}_{n}^{(q)}(R) \mathrm{d} R
\end{aligned}
$$

\subsection{Factored density matrix}

In order to derive a matrix representation of the master equation (7) that is amenable to an efficient solution with methods for PDEs provided that the probabilistic operator $\hat{\rho}(\tau)$ is given at the initial time $\tau$, I split $\hat{H}_{\mathrm{eff}}=\hat{T}_{\mathrm{di}}^{\prime}+\hat{W}[$ Eq. (8)] with $\hat{T}_{\mathrm{di}}^{\prime}=\int_{0}^{\infty} \hat{T}_{\mathrm{di}} \otimes|R\rangle_{\mathrm{NN}}\langle R| \mathrm{d} R[$ [Eq. (33) $]$. Then the matrix representation of Eqs. (19), (20), and (21) becomes

$$
\begin{aligned}
\frac{\partial \rho_{m n}^{(p)}\left(R, R^{\prime}, t\right)}{\partial t}= & \frac{\mathrm{i}}{2 \mu}\left(\frac{1}{R} \frac{\partial^{2}}{\partial R^{2}} R-\frac{1}{R^{\prime}} \frac{\partial^{2}}{\partial R^{\prime 2}} R^{\prime}\right) \rho_{m n}^{(p)}\left(R, R^{\prime}, t\right)-\mathrm{i} \sum_{\ell \in \mathbb{A}_{\mathrm{st}}^{(p)}} W_{m \ell}^{(p)}(R, t) \rho_{\ell n}^{(p)}\left(R, R^{\prime}, t\right) \\
& +\mathrm{i} \sum_{\ell \in \mathbb{A}_{\mathrm{st}}^{(p)}} \rho_{m \ell}^{(p)}\left(R, R^{\prime}, t\right) W_{n \ell}^{(p) *}\left(R^{\prime}, t\right)+\delta\left(R-R^{\prime}\right) \delta_{m n} \sum_{q=p}^{N} \sum_{\ell \in \mathbb{A}_{\mathrm{st}}^{(q)}} \gamma_{m \ell}^{(p q)}(R, t) \rho_{\ell \ell}^{(q)}(R, R, t)
\end{aligned}
$$


where the first summand on the right-hand side stems from the Hermitian $\hat{T}_{\mathrm{di}}^{\prime}$. Moreover, I inserted the completeness relation (30) to transform the operator products $\hat{W} \hat{\rho}(t)$ and $\hat{\rho}(t) \hat{W}^{\dagger}$ in (7) giving the second and third summands in (42); the matrix elements of $\hat{W}$ are

$$
\begin{aligned}
& \mathrm{E}\left\langle p ; m R\left|\otimes_{\mathrm{N}}\left\langle R|\hat{W}| q ; n R^{\prime}\right\rangle_{\mathrm{E}} \otimes\right| R^{\prime}\right\rangle_{\mathrm{N}} \\
= & \delta\left(R-R^{\prime}\right) \delta_{p q} W_{m n}^{(p)}(R, t) \\
= & \delta\left(R-R^{\prime}\right) \delta_{p q}\left[\delta_{m n}\left(E_{m}^{(p)}(R)-\frac{\mathrm{i}}{2} \Gamma_{m}^{(p)}(R, t)\right)\right. \\
& \left.+\wp_{m n}^{(p)}(R, t)\right] .
\end{aligned}
$$

The fourth summand in (42) stems from the matrix elements ${ }_{\mathrm{E}}\left\langle p ; m R\left|\otimes \mathrm{N}_{\mathrm{N}}\left\langle R\left|\hat{\mathcal{L}}_{\mathrm{Jp}}[\hat{\rho}(t)]\right| q ; n R^{\prime}\right\rangle_{\mathrm{E}} \otimes\right| R^{\prime}\right\rangle_{\mathrm{N}}$ of the quantum jump superoperator (41) considering (21) to restrict the sum over charge states.

Equation (42) could, in principle be used-by discretizing the continuous variables $R$ and $R^{\prime}$ - to calcu- late $\rho_{m n}^{(p)}\left(R, R^{\prime}, t\right)$. Yet this leads to an enormously high-dimensional matrix equation which is in practice intractable for all but the simplest systems. But $\hat{H}$ does not couple states with different internuclear distance and neither do the transition rates in $\hat{\mathcal{L}}_{\mathrm{Ld}}[\hat{\rho}(t)]$ [Eqs. (3), (39), and (41)], i.e., all matrix representations but $\rho_{m n}^{(p)}\left(R, R^{\prime}, t\right)$ are local in the internuclear distance. Hence I make the product ansatz

$$
\rho_{m n}^{(p)}\left(R, R^{\prime}, t\right)=\frac{\varsigma_{m n}^{(p)}(R, t)}{R} \frac{\varsigma_{m n}^{(p) *}\left(R^{\prime}, t\right)}{R^{\prime}}
$$

for the density matrix. Substituting this into expression (42) leads-by multiplying with $R R^{\prime}$, integrating over $R^{\prime}$, and dividing by $\tilde{\varsigma}_{m n}^{(p) *}(t)=$ $\int_{0}^{\infty} \varsigma_{m n}^{(p) *}\left(R^{\prime}, t\right) \mathrm{d} R^{\prime}$, if nonzero- to the decoupled equation

$$
\begin{aligned}
\frac{\partial \varsigma_{m n}^{(p)}(R, t)}{\partial t}= & \frac{\mathrm{i}}{2 \mu} \frac{\partial^{2}}{\partial R^{2}} \varsigma_{m n}^{(p)}(R, t)+U_{m n}^{(p)}(R, t)-\mathrm{i} \sum_{\ell \in \mathbb{A}_{\mathrm{st}}^{(p)}} W_{m \ell}^{(p)}(R, t) \varsigma_{\ell n}^{(p)}(R, t) \\
& +\frac{\delta_{m n}}{\tilde{\varsigma}_{m m}^{(p) *}(t)} \sum_{q=p}^{N} \sum_{\ell \in \mathbb{A}_{\mathrm{st}}^{(q)}} \gamma_{m \ell}^{(p q)}(R, t)\left|\varsigma_{\ell \ell}^{(q)}(R, t)\right|^{2},
\end{aligned}
$$

with the complex potential

$$
\begin{aligned}
U_{m n}^{(p)}(R, t)= & -\frac{1}{\tilde{\varsigma}_{m n}^{(p) *}(t)}\left[\varsigma_{m n}^{(p)}(R, t)\left(\frac{\partial \tilde{\varsigma}_{m n}^{(p) *}(t)}{\partial t}+\frac{\mathrm{i}}{2 \mu} \int_{0}^{\infty} \frac{\partial^{2}}{\partial R^{\prime 2}} \varsigma_{m n}^{(p) *}\left(R^{\prime}, t\right) \mathrm{d} R^{\prime}\right)\right. \\
& \left.-\mathrm{i} \sum_{\ell \in \mathbb{A}_{\mathrm{st}}^{(p)}} \varsigma_{m \ell}^{(p)}(R, t) \int_{0}^{\infty} \varsigma_{m \ell}^{(p) *}\left(R^{\prime}, t\right) W_{n \ell}^{(p) *}\left(R^{\prime}, t\right) \mathrm{d} R^{\prime}\right] .
\end{aligned}
$$

The factorization of the density matrix (44) has broken down the problem of calculating $\varsigma_{m n}^{(p)}(R, t)$ to a equation that only depends on a single internuclear distance which is numerically tractable.

\subsection{Quantum Monte Carlo formalism}

Here I specialize the quantum Monte Carlo algorithm from Sect. 2.4 to dissociating molecules. Given a set $\mathbb{S}$ of states at the initial time $\tau$, non-Hermitian propagation for $\Psi \in \mathbb{S}$ is governed by equations of motion (EOMs) which are obtained within the framework of MCWP from the time-dependent Schrödinger equation (15) with the ef fective Hamiltonian (8) by making the ansatz

$$
|\Psi, t\rangle=\sum_{q \in \mathbb{A}_{\mathrm{el}}} \sum_{n \in \mathbb{A}_{\mathrm{st}}^{(q)}} \int_{0}^{\infty} \frac{\varphi_{n}^{(q)}(R, t)}{R}|q ; n R\rangle_{\mathrm{E}} \otimes|R\rangle_{\mathrm{N}} \mathrm{d} R,
$$

for the wave packet of the electronic-distortional motion. The expansion coefficients are the nuclear wave functions $\frac{\varphi_{n}^{(q)}(R, t)}{R}$ where a division by $R$ is made explicitly in order to simplify the resulting EOMs for the nuclear dynamics. The EOMs for the quantum dynamics in a specific charge state follow [24, 25, 26, 27, 28, 29, 30, 31] by inserting the wave packet (47) and the effective Hamiltonian (8), (31), and (39) into the timedependent Schrödinger equation (15) and projecting onto the basis state ${ }_{\mathrm{E}}\langle p ; m R| \otimes{ }_{\mathrm{N}}\langle R|$ giving 


$$
\begin{aligned}
\mathrm{i} \frac{\partial}{\partial t} \varphi_{m}^{(p)}(R, t) & =\sum_{q \in \mathbb{A}_{\mathrm{el}}} \sum_{n \in \mathbb{A}_{\mathrm{st}}^{(q)}} \int_{0}^{\infty} \mathrm{E}\left\langle p ; m R\left|\otimes{ }_{\mathrm{N}}\left\langle R\left|R \hat{H}_{\mathrm{eff}} \frac{\varphi_{n}^{(q)}\left(R^{\prime}, t\right)}{R^{\prime}}\right| q ; n R^{\prime}\right\rangle_{\mathrm{E}} \otimes\right| R^{\prime}\right\rangle_{\mathrm{N}} \mathrm{d} R^{\prime} \\
& =\left[-\frac{1}{2 \mu} \frac{\partial^{2}}{\partial R^{2}}+E_{m}^{(p)}(R)-\frac{\mathrm{i}}{2} \Gamma_{m}^{(p)}(R, t)\right] \varphi_{m}^{(p)}(R, t)-\sum_{n \in \mathbb{A}_{\mathrm{st}}^{(q)}} \wp_{m n}^{(p)}(R, t) \varphi_{n}^{(p)}(R, t) .
\end{aligned}
$$

The EOMs (48) form a linear system of PDEs 56] for the nuclear wave functions which are first-order in the time derivative and second-order in the spatial derivative. Prior to the occurrence of any quantum jumps in the QMC algorithm of Sect. 2.4.2, the summand in the trajectoryaverage for the density operator [confer Eq. [25] ] is the outer product $|\Psi, t\rangle\langle\Psi, t|$ of (47) describing only the neutral molecule. The probability for quantum jumping is then derived from (13) via (36) to

$\delta p_{\Psi, n m}^{(q p)}(t)=\left.\delta t \int_{0}^{\infty} \gamma_{n m}^{(q p)}(R, t)\right|_{\mathrm{E}}\left\langle p ; m R\left|\otimes_{\mathrm{N}}\langle R \mid \Psi, t\rangle\right|^{2} \mathrm{~d} R\right.$.

The expansion coefficients are given by ${ }_{\mathrm{E}}\langle p ; m R| \otimes$ ${ }_{\mathrm{N}}\langle R \mid \Psi, t\rangle=\frac{\varphi_{m}^{(p)}(R, t)}{R}$. Note the integration over $R$ in the equation; for a direct translation with (36), it would be missing. Doing so is reminiscent of Eq. (50) below and acknowledges that quantum jumps shall only take place between discrete indices.

Quantum jumping is mediated by the operator $\hat{J}_{n m}^{(q p)}(R)$ [Eq. (38)] which enters the formulation of the superoperator $\hat{\mathcal{L}}_{\mathrm{Jp}}[\hat{\rho}(t)]$ [Eq. (41)]. Yet this straight translation of the quantum jump operator via (36) to diatomic molecules is unfavorable because it depends on the continuous variable $R \in] 0 ; \infty\left[\right.$. In other words, the $\hat{J}_{n m}^{(q p)}(R)$ promote a Dirac- $\delta$ wave packet. Upon discretization, this implies that there are a huge number of quantum jump operators. To circumvent this situation, I define an integrated quantum jump operator over the internuclear distance via

$$
\hat{\mathfrak{J}}_{n m}^{(q p)}=\int_{0}^{\infty} \hat{J}_{n m}^{(q p)}(R) \mathrm{d} R
$$

This trick removes the dependance of the original quantum jump operator $\hat{J}_{n m}^{(q p)}(R)$ on the continuous variable $R$ and thus only a dependance of $\hat{\mathfrak{J}}_{n m}^{(q p)}$ on discrete variables remains. However, the definition (50) is not motivated by the structure of the equations derived so far and I need to examine how this operator can be put into the expressions to remove their dependence on $\hat{J}_{n m}^{(q p)}(R)$. Equation (50) is introduced in Refs. [24, 25, 26, 27, 28, 29, 30, 31] but not motivated and using the form (50) has consequences unaccounted for therein. Namely, inspecting the first line in (41), I realize that the quantum jump superoperator in terms of the $\hat{\mathfrak{J}}_{n m}^{(q p)}$ has to be expressed as

$$
\begin{aligned}
\hat{\mathcal{L}}_{\mathrm{Jp}}[\hat{\rho}(t)]= & \sum_{p, q \in \mathbb{A}_{\mathrm{el}}} \sum_{m \in \mathbb{A}_{\mathrm{st}}^{(p)}} \sum_{n \in \mathbb{A}_{\mathrm{st}}^{(q)}} \int_{0}^{\infty} \hat{Q}(R) \hat{\mathfrak{J}}_{n m}^{(q p)} \\
& \times \hat{\rho}(t) \hat{\mathfrak{J}}_{n m}^{(q p) \dagger} \hat{Q}(R) \mathrm{d} R
\end{aligned}
$$

with $\hat{Q}(R)=\hat{\mathbb{1}}_{\mathrm{E}} \otimes|R\rangle_{\mathrm{N} N}\langle R|=\hat{Q}^{\dagger}(R)$, where $\hat{\mathbb{1}}_{\mathrm{E}}$ is the unit operator in the direct sum Hilbert space of the electronic states. Clearly, I have $\hat{Q}(R) \hat{\mathfrak{J}}_{n m}^{(q p)}=\hat{J}_{n m}^{(q p)}(R)$ and Eq. (51) implies that $\hat{\mathfrak{J}}_{n m}^{(q p)}|\Psi, t\rangle\langle\Psi, t| \hat{\mathfrak{J}}_{n m}^{(q p) \dagger}$ is not a term in the sums therein. Put differently, expressing (41) by replacing $\hat{J}_{n m}^{(q p)}(R)$ with $\hat{\mathfrak{J}}_{n m}^{(q p)}$ gives incorrect equations.

A quantum jump mediated by (50) can no longer be expressed as an operation on a state vector as before (23) but has to be written as a density operator. Let the state vector at $t+\delta t$ for fixed quantum numbers $p, q \in \mathbb{A}_{\mathrm{el}}$, $m \in \mathbb{A}_{\mathrm{st}}^{(p)}$, and $n \in \mathbb{A}_{\mathrm{st}}^{(q)}$, be the promoted wave packet

$$
\begin{aligned}
|\Psi, t+\delta t\rangle= & \sqrt{\frac{\delta t}{\delta p_{\Psi, n m}^{(q p)}(t)}} \hat{\mathfrak{J}}_{n m}^{(q p)}|\Psi, t\rangle \\
= & \sqrt{\frac{\delta t}{\delta p_{\Psi, n m}^{(q p)}(t)}} \int_{0}^{\infty} \sqrt{\gamma_{n m}^{(q p)}(R, t)} \frac{\varphi_{m}^{(p)}(R, t)}{R} \\
& \times|q ; n R\rangle_{\mathrm{E}} \otimes|R\rangle_{\mathrm{N}} \mathrm{d} R
\end{aligned}
$$

which is normalized, i.e., $\langle\Psi, t+\delta t \mid \Psi, t+\delta t\rangle=1$. With this the probabilistic operator reads

$$
\hat{\varrho}(t+\delta t)=\int_{0}^{\infty} \hat{Q}(R)|\Psi, t+\delta t\rangle\langle\Psi, t+\delta t| \hat{Q}(R) \mathrm{d} R
$$

its trace is unity, i.e., $\operatorname{Tr} \varrho(t+\delta t)=\langle\Psi, t+\delta t \mid \Psi, t+\delta t\rangle=1$

The promoted wave packet (52) is the basis for the next cycle in the Monte Carlo algorithm. The $\hat{\varrho}(t+\delta t)$ is the result of a quantum jump occurring at $t$. For the next time step from $t+\delta t$ to $t+2 \delta t$, I insert $\hat{\varrho}(t+\delta t)$ into the short-time master equation (10): 


$$
\begin{aligned}
\hat{\varrho}(t+2 \delta t)= & \hat{\varrho}(t+\delta t)-\mathrm{i} \delta t \int_{0}^{\infty} \hat{Q}(R)\left(\hat{H}_{\mathrm{eff}}|\Psi, t+\delta t\rangle\langle\Psi, t+\delta t|-| \Psi, t+\delta t\rangle\langle\Psi, t+\delta t| \hat{H}_{\mathrm{eff}}^{\dagger}\right) \hat{Q}(R) \mathrm{d} R \\
& +\delta t \int_{0}^{\infty} \hat{Q}(R)\left(\sum_{p, q \in \mathbb{A}_{\mathrm{el}}} \sum_{m \in \mathbb{A}_{\mathrm{st}}^{(p)}} \sum_{n \in \mathbb{A}_{\mathrm{st}}^{(q)}} \hat{\mathfrak{J}}_{n m}^{(q p)}|\Psi, t+\delta t\rangle\langle\Psi, t+\delta t| \hat{\mathfrak{J}}_{n m}^{(q p) \dagger}\right) \hat{Q}(R) \mathrm{d} R+O\left(\delta t^{2}\right) .
\end{aligned}
$$

Here I exploit the commutators $\left[\hat{H}_{\text {eff }}, \hat{Q}(R)\right]=$ $\left[\hat{H}_{\mathrm{eff}}^{\dagger}, \hat{Q}(R)\right]=0$. Further inserting (53) into (51) is the same as using the outer product instead; the superoperator (51) already contains a projection with $\hat{Q}(R)$. Thereby, I use $\hat{Q}(R) \hat{Q}\left(R^{\prime}\right)=\delta\left(R-R^{\prime}\right) \hat{Q}(R)$ and the commutators $\left[\hat{\mathfrak{J}}_{n m}^{(q p)}, \hat{Q}(R)\right]=\left[\hat{\mathfrak{J}}_{n m}^{(q p) \dagger}, \hat{Q}(R)\right]=0$ and replace $\left[\delta\left(R-R^{\prime}\right)\right]^{2}$ by $\delta\left(R-R^{\prime}\right)$. Equation (54) corresponds to a solution of (10) with $|\Psi, t+\delta t\rangle\langle\Psi, t+\delta t|$ and a projection of its result at time $t+2 \delta t$ with $\hat{Q}(R)$ thereafter, i.e., performing an operation as in (53). Based on this finding, I use state vectors as before and either propagate (48) or quantum jump (51); the result, however, then needs to be projected (53) to be meaningful.

The probabilities for quantum jumping - after an initial quantum jump has occurred - need to be derived for the probabilistic operator (53). I use the trace condition (11) for the quantum jump superoperator (51) giving

$$
\delta p_{\Psi}(t)=\delta t \operatorname{Tr} \hat{\mathcal{L}}_{\mathrm{Jp}}[\hat{\varrho}(t)]=\sum_{p, q \in \mathbb{A}_{\mathrm{el}}} \sum_{m \in \mathbb{A}_{\mathrm{st}}^{(p)}} \sum_{n \in \mathbb{A}_{\mathrm{st}}^{(q)}} \delta p_{\Psi, n m}^{(q p)}(t)
$$

This leads to the same expression (49) for $\delta p_{\Psi, n m}^{(q p)}(t)$ as before.

The upshot is that one can solve the master equation by Monte Carlo wave packet propagation with (50) along the lines of Sect. 2.4.2 where, however, a projection (53) is required henceforth after the first quantum jump has occurred. Let me go through the algorithm of Sect. 2.4.2 in detail. The first step selects the starting state vector and is unmodified. The second step uses the probability (49) to decide whether to quantum jump or not. The third step uses (48) to temporally propagate. The fourth step quantum jumps employing (52). The density operator of the system is then approximated analogously to (25) where, however, the outer products are replaced by (53) once a quantum jump has happened.

\section{Conclusion}

Molecules in ultrafast and intense light exhibit a fascinating phenomenology of intertwined electronic and nuclear quantum dynamics involving (multiple) ionization, radiative and electronic decay, rotation, vibration and dissociation. To tackle this situation, I devise a rigorous quantum optical formalism, initially in abstract form, centered around the master equation in Lindblad form. It describes the time evolution of the probabilistic operator for system-reservoir interaction using the Markoff approximation allowing for the dissipation of particles and energy. The system, thereby, is the dissociating molecule which dissipates photons and electrons into the reservoir, i.e., the continuum. A short-time approximation of the master equation is derived. In a direct sum of rigged Hilbert spaces for multiple charge states, I reduce it to a hierarchy of equations. This allows me to solve them using methods for systems of differential equations. Alternatively, quantum Monte Carlo wave packet propagation (MCWP) is proposed that yields the density operator by averaging over an ensemble of quantum trajectories. The MCWP approach is computationally more efficient than a numerical integration of the master equation and facilitates a lucid physical interpretation of the resulting quantum trajectories in terms of propagation and quantum jumps for a single state vector. The abstract formalism is concretized to diatomic molecules in Born-Oppenheimer approximation. The basis states are the electronic-distortional states for a fixed-in-space intermolecular axis, i.e., molecular rotation is disregarded. An equation for a factored density matrix which depends only on a single internuclear distance is derived. It is amenable to a solution with methods for PDEs. The MCWP formulation requires to put special emphasize on quantum jumps whereby an integrated jump operator is used. This, however, necessitates the use of a projection afterwards which has incorrectly been omitted in Refs. [24, 25, 26, 27, 28, 29, 30, 31], i.e., a density operator description is necessay. Forthcoming computational work based on the present formalism shall uncover the impact of the correct treatment of quantum jumps put forward here in comparison to Refs. [24, 25, 26, 27, 28, 29, 30, 31].

Deterministic sampling has been proposed to solve the master equation for a small number of potential energy surfaces (PES) considered [24, 25, 26, 27, 28, 29, 30, 31]. This is a complementary notion to the solution of the hierarchy of equations for different charge states put forward here. In the specialized case of no coupling of electronic states with the same number of electrons by the quantum jump part of the Lindblad superoperator - that is exclusively considered in Refs. [24, 25, 26, 27, 28, 29, 30, 31]probability theory can be used. Starting from a propagation on a single ground-state PES quantum jumps to other states are made in sufficient frequency in order to sample these transitions adequately for the quantities of interest to be converged, e.g., the kinetic energy release. 
This prescription is a formally exact probabilistic solution. However, there is also a weakness of a strong increase of the number of possible pathways. Namely, the groundstate wave packets need to be propagated for all times and quantum jumps are applied to all accessible destinations with certain frequency from whence a further propagation is necessary. In the light of this, I conclude that deterministic sampling does not seem to provide any advantage over the numerical integration of the master equation. On the contrary, only a single solution of the PDEs is required whereas multiple propagations are required for higher charge states in deterministic sampling.

The presented quantum optical formalism is very basic and may find ubiquitous use to describe the quantum dynamics of light-matter interaction. It thus provides manifold prospects for future research. A particular feature of the presented approach is that ionization processes which reduce the number of electrons are accounted for solely by transition rates and the outgoing electron itself is not regarded "it just disappears." If also the electron dynamics shall be included, some modifications are in order using a complex absorbing potential to absorb the electron [65].

So far the MCWP method has only been applied in the context of the photoionization of diatomic molecules [24, 25, 26, 27, 28, 29, 30, 31] in the optical and the XUV regime. An extension to the x-ray domain, where radiative and electronic decay become manifest [1, 2, 3, 4, 4], is a fascinating and highly pressing issue. Thereby, not only electronic decay involving core holes may be relevant but there are also situations in which inner-valence vacancies undergo an ultrafast electronic decay [66, 13].

The formalism of Sect. 2 and Sect. 3 is also directly applicable to atoms. To this end, I simply omit the internuclear distance in the basis states and modify the equations appropriately. The MCWP is a step ahead over a Monte Carlo solution of rate equations for atoms [67, 68] as also coherent phenomena are describeable.

Molecular rotation is not described in this work because it only leads to a small energy splitting compared with the electronic-distortional motion. In future work, one may consider a treatment of molecular rotations, specifically rotational excitation by electronic transitions and nuclear distortion and molecular alignment by the light [55]. Here the consequences of the nuclear expansion (flexible rotor) [59] of a dissociating molecule on the rotation spectra is to be treated thoroughly.

X-ray quantum optics 69 comes into reach with molecules; a number of studies have been conducted for atoms which now await an examination for molecules facilitated by the present article. This bears a high potential for discovery that goes beyond what can be achieved with rate equations $3,44,49,50,51$. Namely, if the x-ray energy is tuned to a resonance, then coherences manifest. Especially the two-color physics of FEL $x$ rays and an optical laser offers further promising avenues 69].

I have been mostly concerned with XUV light and $\mathrm{x}$ rays from FELs here but attosecond light sources offer exciting new possibilities for studying molecules and the presented theory shall be applicable in this situation as well. Such sources are particularly interesting because they offer a much finer control of the beam characteristics compared with present-day FELs.

\section{Acknowledgments}

I am grateful to Mathias Nest for thoughtful discussions. So I am to Lorenz S. Cederbaum and Jochen Schirmer and I thank them for a critical reading of the manuscript. This research did not receive any specific grant from funding agencies in the public, commercial, or not-for-profit sectors. Declarations of interest: none.

\section{References}

\section{References}

[1] M. Hoener, L. Fang, O. Kornilov, O. Gessner, S. T. Pratt, M. Gühr, E. P. Kanter, C. Blaga, C. Bostedt, J. D. Bozek, P. H. Bucksbaum, C. Buth, M. Chen, R. Coffee, J. Cryan, L. DiMauro, M. Glownia, E. Hosler, E. Kukk, S. R. Leone, B. McFarland, M. Messerschmidt, B. Murphy, V. Petrovic, D. Rolles, N. Berrah, Ultraintense x-ray induced ionization, dissociation, and frustrated absorption in molecular nitrogen, Phys. Rev. Lett. 104 (2010) 253002. doi:10.1103/PhysRevLett.104.253002.

[2] L. Fang, T. Osipov, B. Murphy, F. Tarantelli, E. Kukk, J. Cryan, P. H. Bucksbaum, R. N. Coffee, M. Chen, C. Buth, N. Berrah, Multiphoton ionization as a clock to reveal molecular dynamics with intense short x-ray free electron laser pulses, Phys. Rev. Lett. 109 (2012) 263001. arXiv:1301.6459, doi:10.1103/PhysRevLett.109.263001.

[3] C. Buth, J.-C. Liu, M. H. Chen, J. P. Cryan, L. Fang, J. M. Glownia, M. Hoener, R. N. Coffee, N. Berrah, Ultrafast absorption of intense $\mathrm{x}$ rays by nitrogen molecules, J. Chem. Phys. 136 (2012) 214310. arXiv:1201.1896, doi:10.1063/1.4722756

[4] J.-C. Liu, N. Berrah, L. S. Cederbaum, J. P. Cryan, J. M. Glownia, K. J. Schafer, C. Buth, Rate equations for nitrogen molecules in ultrashort and intense $\mathrm{x}$-ray pulses, J. Phys. B 49 (2016) 075602. arXiv:1508.05223, doi:10.1088/0953-4075/49/7/075602.

[5] Y. Hao, L. Inhester, K. Hanasaki, S.-K. Son, R. Santra, Efficient electronic structure calculation for molecular ionization dynamics at high x-ray intensity, Struct. Dyn. 2 (2015) 041707. doi:10.1063/1.4919794

[6] L. Inhester, K. Hanasaki, Y. Hao, S.-K. Son, R. Santra, X-ray multiphoton ionization dynamics of a water molecule irradiated by an x-ray free-electron laser pulse, Phys. Rev. A 94 (2016) 023422. doi:10.1103/PhysRevA.94.023422

[7] A. Rudenko, L. Inhester, K. Hanasaki, X. Li, S. J. Robatjazi, B. Erk, R. Boll, K. Toyota, Y. Hao, O. Vendrell, C. Bomme, E. Savelyev, B. Rudek, L. Foucar, S. H. Southworth, C. S. Lehmann, B. Kraessig, T. Marchenko, M. Simon, K. Ueda, K. R. Ferguson, M. Bucher, T. Gorkhover, S. Carron, R. AlonsoMori, J. E. Koglin, J. Correa, G. J. Williams, S. Boutet, L. Young, C. Bostedt, S.-K. Son, R. Santra, D. Rolles, Femtosecond response of polyatomic molecules to ultra-intense hard X-rays, Nature 546 (2017) 129-132. doi:10.1038/nature22373.

[8] D. J. Tannor, Introduction to Quantum Mechanics: A TimeDependent Perspective, University Science Books, Sausalito, California, 2007.

[9] L. S. Cederbaum, F. Tarantelli, Nuclear dynamics of decaying states: A time-dependent formulation, J. Chem. Phys. 98 (1993) 9691-9706. doi:10.1063/1.464348 
[10] L. S. Cederbaum, F. Tarantelli, Nuclear dynamics of several decaying overlapping electronic states: A timedependent formulation, J. Chem. Phys. 99 (1993) 5871-5884. doi:10.1063/1.465940

[11] E. Pahl, L. S. Cederbaum, F. Tarantelli, Resonant decay spectra for energetically unselective excitation exemplified by the broadband resonant Auger spectrum of HF, Phys. Rev. A 60 (1999) 1070-1078. doi:10.1103/PhysRevA.60.1070

[12] E. Pahl, J. Brand, L. S. Cederbaum, F. Tarantelli, Impact of narrow-band excitation on resonant decay spectra, Phys. Rev. A 60 (1999) 1079-1090. doi:10.1103/PhysRevA.60.1079

[13] S. Scheit, L. S. Cederbaum, H.-D. Meyer, Time-dependent interplay between electron emission and fragmentation in the interatomic Coulombic decay, J. Chem. Phys. 118 (2003) 2092-2107. doi: $10.1063 / 1.1531996$

[14] L. S. Cederbaum, Y.-C. Chiang, P. V. Demekhin, N. Moiseyev, Resonant Auger decay of molecules in intense x-ray laser fields: light-induced strong nonadiabatic effects, Phys. Rev. Lett. 106 (2011) 123001. doi:10.1103/PhysRevLett.106.123001

[15] N. Sisourat, Nuclear dynamics of decaying states: A semiclassical approach, J. Chem. Phys. 139 (2013) 074111. doi:10.1063/1.4818491

[16] J. M. Glownia, J. Cryan, J. Andreasson, A. Belkacem, N. Berrah, C. I. Blaga, C. Bostedt, J. Bozek, L. F. DiMauro, L. Fang, J. Frisch, O. Gessner, M. Gühr, J. Hajdu, M. P. Hertlein, M. Hoener, G. Huang, O. Kornilov, J. P. Marangos, A. M. March, B. K. McFarland, M. H., V. S. Petrovic, C. Raman, D. Ray, D. A. Reis, M. Trigo, J. L. White, W. White, R. Wilcox, L. Young, R. N. Coffee, P. H. Bucksbaum, Timeresolved pump-probe experiments at the LCLS, Opt. Express 18 (2010) 17620-17630. doi:10.1364/0E.18.017620

[17] A. M. Hanna, O. Vendrell, A. Ourmazd, R. Santra, Laser control over the ultrafast Coulomb explosion of $\mathrm{N}_{2}^{2+}$ after Auger decay: A quantum-dynamics investigation, Phys. Rev. A 95 (2017) 043419. doi:10.1103/PhysRevA.95.043419

[18] G. Lindblad, On the generators of quantum dynamical semigroups, Comm. Math. Phys. 48 (1976) 119-130. doi: 10.1007/BF01608499

[19] W. H. Louisell, Quantum Statistical Properties of Radiation, Wiley Classics Library, John Wiley \& Sons, New York, 1990.

[20] K. Blum, Density Matrix Theory and Applications, 2nd Edition, Physics of Atoms and Molecules, Plenum Press, New York, 1996. doi:10.1007/978-1-4757-4931-1

[21] M. O. Scully, M. S. Zubairy, Quantum Optics, Cambridge University Press, Cambridge, New York, Melbourne, 1997. doi:10.1017/CB09780511813993

[22] P. Meystre, M. Sargent III, Elements of Quantum Optics, 3rd Edition, Springer, Berlin, 1999. doi:10.1007/978-3-662-03877-2

[23] C. W. Gardiner, P. Zoller, Quantum Noise, 3rd Edition, Springer Series in Synergetics, Springer, Berlin, Heidelberg, New York, 2004.

[24] H. A. Leth, Multiple ionization processes in diatomic molecules exposed to short intense laser pulses - The Monte Carlo wave packet approach, Master thesis, Aarhus Universitet, Institut for Fysik og Astronomi, Ny Munkegade 120, 8000 Aarhus C, Denmark (May 2009).

URL http://phys.au.dk/fileadmin/site_files/forskning/ltc/thes es/dela4henriette.pdf

[25] H. A. Leth, L. B. Madsen, K. Mølmer, Monte Carlo wave packet theory of dissociative double ionization, Phys. Rev. Lett. 103 (2009) 183601. doi:10.1103/PhysRevLett.103.183601

[26] H. A. Leth, L. B. Madsen, K. Mølmer, Monte Carlo wave packet approach to dissociative multiple ionization in diatomic molecules, Phys. Rev. A 81 (2010) 053409. doi:10.1103/PhysRevA.81.053409

[27] H. A. Leth, L. B. Madsen, K. Mølmer, Dissociative double ionization of $\mathrm{H}_{2}$ and $\mathrm{D}_{2}$ : Comparison between experiment and Monte Carlo wave packet calculations, Phys. Rev. A 81 (2010) 053410. doi:10.1103/PhysRevA.81.053410

[28] H. A. Leth, L. B. Madsen, Dissociative multiple ion- ization of diatomic molecules by extreme-ultraviolet freeelectron-laser pulses, Phys. Rev. A 83 (2011) 063415. doi:10.1103/PhysRevA.83.063415

[29] H. A. Leth, Dissociative ionization. A study using the Monte Carlo wave packet approach, PhD thesis, Aarhus Universitet, Institut for Fysik og Astronomi, Ny Munkegade 120, 8000 Aarhus C, Denmark (jun 2011).

URL http://phys.au.dk/fileadmin/site_files/publikationer/phd /Henriette_Leth.pdf

30] Q. Jing, Monte Carlo wave packet approach to dissociative double ionization of $\mathrm{H}_{2}$ interacting with intense laser pulses, Master thesis, Aarhus Universitet, Institut for Fysik og Astronomi, Ny Munkegade 120, 8000 Aarhus C, Denmark (December 2016). URL http://phys.au.dk/fileadmin/site_files/forskning/ltc/thes es/del_AQingli.pdf

[31] Q. Jing, L. B. Madsen, Laser-induced dissociative ionization of $\mathrm{H}_{2}$ from the near-infrared to the mid-infrared regime, Phys. Rev. A 94 (2016) 063402. doi:10.1103/PhysRevA.94.063402.

[32] J. Dalibard, Y. Castin, K. Mølmer, Wave-function approach to dissipative processes in quantum optics, Phys. Rev. Lett. 68 (1992) 580-583. doi:10.1103/PhysRevLett.68.580

[33] K. Mølmer, Y. Castin, J. Dalibard, Monte Carlo wave-function method in quantum optics, J. Opt. Soc. Am. B 10 (1993) 524538. doi:10.1364/JOSAB.10.000524

[34] K. Mølmer, Y. Castin, Monte Carlo wavefunctions in quantum optics, Quantum Semiclass. Opt. 8 (1996) 49-72. doi:10.1088/1355-5111/8/1/007

[35] M. B. Plenio, P. L. Knight, The quantum-jump approach to dissipative dynamics in quantum optics, Rev. Mod. Phys. 70 (1998) 101-144. doi:10.1103/RevModPhys.70.101

[36] H. Fukuzawa, K. Motomura, X.-J. Liu, G. Prümper, M. Okunishi, K. Ueda, N. Saito, H. Iwayama, K. Nagaya, M. Yao, M. Nagasono, A. Higashiya, M. Yabashi, T. Ishikawa, H. Ohashi, H. Kimura, Ion momentum spectroscopy of $\mathrm{N}_{2}$ and $\mathrm{O}_{2}$ molecules irradiated by EUV free-electron laser pulses, J. Phys. B 42 (2009) 181001. doi:10.1088/0953-4075/42/18/181001

[37] M. H. Beck, A. Jäckle, G. A. Worth, H.-D. Meyer, The multiconfiguration time-dependent Hartree (MCTDH) method: a highly efficient algorithm for propagating wavepackets, Phys. Rep. 324 (2000) 1-105. doi:10.1016/S0370-1573(99) 00047-2

[38] R. de la Madrid, The role of the rigged Hilbert space in quantum mechanics, Eur. J. Phys. 26 (2005) 287-312. doi:10.1088/0143-0807/26/2/008

[39] A. Bohm, H. Uncu, S. Komy, A brief survey of the mathematics of quantum physics, Rep. Math. Phys. 64 (2009) 5-32. doi:10.1016/S0034-4877(09) 00020-2

[40] M. S. Tsalenko (originator), Direct sum, Encyclopedia of Mathematics. Accessed 28 May 2017.

URL http://www.encyclopediaofmath.org/index.php?title=Di rect_sum\&oldid $=34851$

[41] H.-D. Meyer, U. Manthe, L. S. Cederbaum, The multiconfigurational time-dependent Hartree approach, Chem. Phys. Lett. 165 (1990) 73-78. doi:10.1016/0009-2614(90)87014-I.

[42] U. Manthe, H. D. Meyer, L. S. Cederbaum, Wave-packet dynamics within the multiconfiguration Hartree framework: General aspects and application to NOCl, J. Chem. Phys. 97 (1992) 3199-3213. doi:10.1063/1.463007

[43] A. Szabo, N. S. Ostlund, Modern Quantum Chemistry: Introduction to Advanced Electronic Structure Theory, 1st, revised Edition, McGraw-Hill, New York, 1989.

[44] D. R. Hartree, The wave mechanics of an atom with a noncoulomb central field. Part I. Theory and methods, Proc. Camb. Phil. Soc. 24 (1928) 89-110. doi:10.1017/S0305004100011919

[45] E. Merzbacher, Quantum mechanics, 3rd Edition, John Wiley \& Sons, New York, 1998

[46] C. Cohen-Tannoudji, B. Diu, F. Laloë, Quantum Mechanics, John Wiley \& Sons, Singapore, 2005.

[47] V. F. Weisskopf, E. P. Wigner, Berechnung der natürlichen Linienbreite auf Grund der Diracschen Lichttheorie, Z. Phys. 63 (1930) 54-73. doi:10.1007/BF01336768

[48] W. Pauli, Über das H-Theorem vom Anwachsen der Entropie 
vom Standpunkt der neueren Quantenmechanik, in: P. Debye (Ed.), Probleme der modernen Physik, S. Hirzel, Leipzig, 1928, pp. 30-45, Arnold Sommerfeld zum 60. Geburtstag, gewidmet von seinen Schülern.

[49] C. Buth, R. Beerwerth, R. Obaid, N. Berrah, L. S. Cederbaum, $\mathrm{S}$. Fritzsche, Neon in ultrashort and intense x rays from freeelectron lasers, J. Phys. B 51 (2018) 055602. arXiv:1705.07521, doi:10.1088/1361-6455/aaa39a

[50] R. Obaid, C. Buth, G. Dakovski, R. Beerwerth, M. Holmes, J. Aldrich, M.-F. Lin, M. Minitti, T. Osipov, W. Schlotter, L. S. Cederbaum, S. Fritzsche, N. Berrah, LCLS inphoton out: fluorescence measurement of neon using soft x-rays, J. Phys. B 51 (2018) 034003. arXiv:1708.01283. doi:10.1088/1361-6455/aaa189

[51] C. Buth, Nonlinearity in the sequential absorption of multiple photons, submitted, arXiv:1612.07105

[52] M. I. Shabunin (originator), Order relation Encyclopedia of Mathematics. Accessed 17 May 2017.

URL http://www.encyclopediaofmath.org/index.php?title=Or der_relation\&oldid $=16014$

[53] I. B. Vapnyarskii (originator), Euler method, Encyclopedia of Mathematics. Accessed 25 May 2017.

URL http://www.encyclopediaofmath.org/index.php?title=Eu ler_method\&oldid $=16352$

[54] V. V. Bobkov (originator), Runge-Kutta method Encyclopedia of Mathematics. Accessed 25 May 2017.

URL http://www.encyclopediaofmath.org/index.php?title=Ru nge-Kutta_method\&oldid=22999

[55] C. Buth, R. Santra, Theory of x-ray absorption by laser-aligned symmetric-top molecules, Phys. Rev. A 77 (2008) 013413. arXiv:0711.3203 doi:10.1103/PhysRevA.77.013413

[56] G. B. Arfken, H. J. Weber, Mathematical Methods for Physicists, 6th Edition, Elsevier Academic Press, New York, 2005.

[57] V. A. Uspenskii (originator), Algorithm Encyclopedia of Mathematics. Accessed 02 April 2017.

URL http://www.encyclopediaofmath.org/index.php?title=Al gorithm\&oldid=18759

[58] L. A. Skornyakov (originator), Order (on a set) Encyclopedia of Mathematics. Accessed 17 May 2017.

URL http://www.encyclopediaofmath.org/index.php?title=Or der_(on_a_set)\&oldid=37418
[59] H. W. Kroto, Molecular Rotation Spectra, John Wiley \& Sons, London, 1975.

[60] P. W. Atkins, R. S. Friedman, Molecular Quantum Mechanics, 4th Edition, Oxford University Press, Oxford, 2004.

[61] H. J. Monkhorst, Chemical physics without the BornOppenheimer approximation: The molecular coupledcluster method, Phys. Rev. A 36 (1987) 1544-1561. doi:10.1103/PhysRevA.36.1544

[62] M. Born, J. R. Oppenheimer, Zur Quantentheorie der Molekeln, Ann. Phys. (Leipzig) 84 (1927) 457-484. doi:10.1002/andp.19273892002

[63] H. Köppel, W. Domcke, L. S. Cederbaum, Multimode molecular dynamics beyond the Born-Oppenheimer approximation, in: I. Prigogine, S. A. Rice (Eds.), Adv. Chem. Phys., Vol. 57, John Wiley \& Sons, Hoboken (New Jersey), 1984, pp. 59-246. doi:10.1002/9780470142813.ch2

[64] R. A. Minlos (originator), Fock space Encyclopedia of Mathematics. Accessed 26 April 2017.

URL http://www.encyclopediaofmath.org/index.php?title=Fo ck_space\&oldid=13259

[65] S. Selst $\varnothing$, S. Kvaal, Absorbing boundary conditions for dynamical many-body quantum systems, J. Phys. B 43 (2010) 065004. doi:10.1088/0953-4075/43/6/065004

[66] C. Buth, R. Santra, L. S. Cederbaum, Impact of interatomic electronic decay processes on the width of the $\mathrm{Xe} 4 d$ lines in the Auger decay of the xenon fluorides, J. Chem. Phys. 119 (2003) 10575-10584. arXiv:physics/0303100. doi:10.1063/1.1620502

[67] S.-K. Son, R. Santra, Monte Carlo calculation of ion, electron, and photon spectra of xenon atoms in x-ray freeelectron laser pulses, Phys. Rev. A 85 (2012) 063415. doi:10.1103/PhysRevA.85.063415.

[68] S.-K. Son, R. Santra, Erratum: Monte Carlo calculation of ion, electron, and photon spectra of xenon atoms in x-ray free-electron laser pulses [Phys. Rev. A 85, 063415 (2012)], Phys. Rev. A 92 (2015) 039906(E). doi:10.1103/PhysRevA.92.039906

[69] B. W. Adams, C. Buth, S. M. Cavaletto, J. Evers, Z. Harman, C. H. Keitel, A. Pálffy, A. Picón, R. Röhlsberger, Y. Rostovtsev, K. Tamasaku, X-ray quantum optics, J. Mod. Opt. 60 (2013) 2-21. doi:10.1080/09500340.2012.752113 